\title{
Feedback dose alteration significantly affects probability of pathogen eradication in nosocomial pneumonia
}

\author{
F. Scaglione*, S. Esposito\#, S. Leone*, V. Lucini*, M. Pannacci*, \\ L. Ma" and G.L. Drusano"
}

ABSTRACT: Nosocomial pneumonia (NP) is associated with considerable morbidity and mortality. Data have shown that inadequate initial antibiotic therapy is a major risk for infectionattributed mortality. The aim of the present study was to measure antibiotic concentration and minimum inhibitory concentration (MIC) in infected hospitalised patients early in therapy, in order to determine whether dose alterations, in those with low drug concentrations, could affect outcomes.

Only patients treated with aminoglycosides, fluoroquinolones, and $\beta$-lactams were evaluated. MICs were determined using standard National Committee for Clinical Laboratory Standards procedures. Antibiotics were assayed using validated high-performance liquid chromatographic methods. Pharmacokinetic/pharmacodynamic markers adopted were: aminoglycoside peak/MIC ratio $\geqslant 8 \mathrm{mg} \cdot \mathrm{L}^{-1}$; fluoroquinolone peak $/ \mathrm{MIC} \geqslant 10 \mathrm{mg} \cdot \mathrm{L}^{-1} ; \beta$-lactam peak $/ \mathrm{MIC} \geqslant 4 \mathrm{mg} \cdot \mathrm{L}^{-1}$ and time that plasma levels remain above the $M I C \geqslant 70 \%$.

638 patients with NP were included in the study. In 205 patients, both drug concentration and isolate MIC were available, while in other patients, used as controls, one or both parameters were lacking. For clinical outcome, the Acute Physiology and Chronic Health Evaluation II score $(p<0.0001)$, the presence of combination therapy $(p=0.0014)$ and whether both MIC and drug concentration(s) were measured $(p=0.0002)$ significantly affected the probability of a good outcome. For microbiological outcome, the MIC for the $\beta$-lactams ( $\left.\leqslant 2 \mathrm{mg} \cdot \mathrm{L}^{-1} ; \mathrm{p}<0.0001\right)$ and whether the second drug was a fluoroquinolone or aminoglycoside (fluoroquinolones were better than aminoglycosides; $\mathrm{p}=\mathbf{0 . 0 1 7 7}$ ), as well as whether both MIC and drug concentration(s) were measured $(p=0.02)$, affected the probability of eradication.

Measurement of drug concentrations and determination of pathogen MIC values with subsequent dose alteration significantly improves the probability of good clinical outcome and pathogen eradication in NP.

KEYWORDS: Nosocomial pneumonia, optimising antibiotic therapy, pharmacokinetic/ pharmacodynamic indices

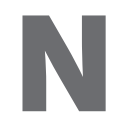
osocomial pneumonia (NP) remains a major cause of mortality and morbidity despite advances in antimicrobial therapy and supportive care [1]. The mortality attributed to an episode of NP is debated but could be as high as 30\% [2]. Multiple studies have shown that NP increases hospital length of stay by an average of 7-10 days and, in patients with ventilator-associated pneumonia, the duration of both mechanical ventilation and intensive care unit stay is increased [3]. In the last $10 \mathrm{yrs}$, evidence has accumulated that initial inappropriate antibiotic treatment is an important independent risk factor for excess mortality in patients with NP. In order to address this problem of excess mortality, several strategies have been suggested [4]. Optimisation of antibiotic dosing regimens is one approach that might ameliorate this problem [5]. Recognising that the minimum inhibitory concentration (MIC) of the pathogen being treated and the individual patient's pharmacokinetic (PK) handling of the drug being employed each have an important and independent impact on the probability of a good clinical and microbiological outcome [6, 7], it becomes crucial to know the MIC of the infecting pathogen for the drug employed and obtain an accurate estimate of the drug exposure to aid in the

\section{AFFILIATIONS}

*Università degli Studi di Milano, Dip. Di Farmacologia, Milano, and \#Dept of Infectious Diseases, Second University of Naples, Naples, Italy. -Ordway Research Institute, Albany, NY, USA.

CORRESPONDENCE

F. Scaglione

Dept of Pharmacology,

Chemotherapy and Toxicology,

Faculty of Medicine

University of Milan

Via Vanvitelli 32

20129 Milan

Italy

E-mail: francesco.scaglione@

unimi.it

Received:

Oct 012008

Accepted after revision:

Jan 212009

First published online:

Feb 122009 
prediction of antimicrobial activity. Unfortunately, in clinical practice, it is rare to be able to know the pharmacokinetics of a drug in a patient [8]. We reasoned that measuring the drug concentrations in infected patients, as well as knowing the infecting organism's MIC, would allow rapid identification of patients at high risk for clinical and microbiological treatment failure. As a primary hypothesis, we wished to test whether measuring drug exposure and pathogen MIC and subsequently altering dose when the seriously infected patient was felt to be at high risk of failure would significantly alter the probability of a good clinical or microbiological outcome.

\section{METHODS}

\section{Study design and population}

We started a PK/pharmacodynamic (PD) programme involving patients with NP. The primary end-point of the programme was to measure $\mathrm{PK} / \mathrm{PD}$ parameters in patients with severe infections and to evaluate the effect on the outcome. Only patients receiving aminoglycosides (amikacin), fluoroquinolones (ciprofloxacin or levofloxacin) and $\beta$-lactams (ceftazidime or cefotaxime) were considered in the study. Acute Physiology and Chronic Health Evaluation (APACHE) II score was calculated in all patients. The sequence of procedures to adjust the dose was the following: 1) isolation of the pathogen and performance of an MIC test, 2) initiation of therapy according to patient's physician choice, 3) PK analysis, 4) adjust dose or interval using PD principles, 5) re-determine concentrations. The adopted PD indices were: aminoglycoside peak/MIC $\geqslant 8 \mathrm{mg} \cdot \mathrm{L}^{-1}$; fluoroquinolone peak/MIC $\geqslant 10 \mathrm{mg} \cdot \mathrm{L}^{-1} ; \beta$-lactam peak/MIC $\geqslant 4 \mathrm{mg} \cdot \mathrm{L}^{-1}$ and time that plasma levels remain above the MIC $(t>$ MIC $) \geqslant 70 \%$. The same values were used for patients receiving monotherapy or combination therapy. For the aminoglycosides and fluoroquinolones, optimising the peak concentration in plasma ( $\mathrm{Cmax}) /$ MIC ratio was the primary objective. While there is literature indicating that the area under the plasma concentration-time curve (AUC)/MIC ratio may be the best PD-linked variable for these agents, we employed the $\mathrm{Cmax} / \mathrm{MIC}$ ratio for tractability of implementation. For $\beta$-lactams, we decided that the $t>$ MIC value should exceed $70 \%$ as the primary goal of therapy. Due to the limited clinical experience with targeting dosing regimens of $\beta$-lactams in this way, maintaining a Cmax/MIC ratio $>4$ was also recommended by our Ethics Committee (Milan, Italy). Only patients treated by intravenous (i.v.) infusion were included in the study. Initial dosage was amikacin $15 \mathrm{mg} \cdot \mathrm{kg}^{-1}$ every $24 \mathrm{~h}$, cefotaxime or ceftazidime $2 \mathrm{~g}$ every $8 \mathrm{~h}$, ciprofloxacin $400 \mathrm{mg}$ every $12 \mathrm{~h}$, and levofloxacin $500 \mathrm{mg}$ every 12 or $24 \mathrm{~h}$. The sampling times used to estimate PK parameters were as follows: aminoglycoside peak at $0.5 \mathrm{~h}$ after end of 30 -min infusion, and fluoroquinolone peak at $0.5 \mathrm{~h}$ after end of 60 -min infusion. $\beta$-lactam sampling times were: peak at $0.5 \mathrm{~h}$ after end of 30-min infusion and then $5.6 \mathrm{~h}$ from start of infusion, which is at $70 \%$ of the 8 -h dosing interval. To estimate dose correction, each patient's data (age, sex, weight, height, serum creatinine and serum albumin), combined with their dosage regimen and respective plasma levels, were analysed using a Bayesian PK approach. In particular, amikacin results were analysed by means of a software package, Abbottbase Pharmacokinetic Systems program, V 1.10, from Abbott Laboratories Diagnostics Division (Abbott Park, IL, USA). Fluoroquinolone results were analysed by means of a software package, ADAPT II (Biomedical Simulations Resource, Los Angeles, CA, USA), using previous PK population data $[9,10]$. Regarding $\beta$-lactams, if applicable, the dosage was changed by increasing either the number of doses (from 2 g every $8 \mathrm{~h}$ to 2 g every $6 \mathrm{~h}$ ) or the infusion time (from $0.5 \mathrm{~h}$ to $3 \mathrm{~h}$ ).

\section{Inclusion and exclusion criteria}

Adult (aged $>18$ yrs) males and females with pneumonia acquired after $48 \mathrm{~h}$ in an inpatient facility were enrolled in this study. Patients had to have at least two of the following: cough, purulent sputum, auscultatory findings of pneumonia, dyspnoea, tachypnoea or hypoxaemia. Patients also had to have at least two of the following: fever or hypothermia, systolic blood pressure $<90 \mathrm{~mm} \mathrm{Hg}$, cardiac frequency $\geqslant 120$ beats $\cdot \mathrm{min}^{-1}$, respiratory frequency $>30$ breaths $\cdot \mathrm{min}^{-1}$, altered mental status, total peripheral white blood cell count $>10,000$ cells $\cdot \mu \mathrm{L}^{-1}$ or $<4,500$ cells $\mu \mathrm{L}^{-1}$ or $>15 \%$ immature neutrophils (band forms), or adequate sputum specimens for Gram stain and culture. Radiographic findings of pneumonia (new or progressive infiltrates, consolidation or pleural effusion) and life expectancy $\geqslant 7$ days were required. Test patients were those in whom the blood levels of the tested antibiotics were available. Patients satisfying all the aforementioned criteria but with no available antibiotic levels and/or infecting pathogens available were used as controls.

Exclusion criteria were: 1) known or suspected meningitis, endocarditis, osteomyelitis, lung cancer or another malignancy metastatic to the lung; 2) cystic fibrosis; 3) suspected active tuberculosis; 4) HIV-positive infection; 5) liver disease and total bilirubin more than five times the upper limit of normal; 6) severe neutropenia $\left(<500\right.$ cells $\left.\cdot \mu \mathrm{L}^{-1}\right)$; and 7$)$ pregnancy. In order to reduce the variability, patients with evidence of sepsis with hypotension and/or end-organ dysfunction, shock, vasopressors required for $>4 \mathrm{~h}$, duration of mechanical ventilation $>5$ days or severe renal impairment requiring dialysis were excluded. Also, since the analysis was confined to third generation cephalosporins, aminoglycosides and fluoroquinolones, patients with staphylococcal infections were excluded.

\section{Data collected}

Demographic variables, such as age, weight and sex, were collected for all patients. APACHE II score was calculated in all patients. The presence or absence of bacteraemia for all patients with NP was recorded.

\section{Isolation of a pathogen from respiratory or blood cultures}

Pathogens were obtained by culture of protected specimen brush sampling or bronchoscopic bronchoalveolar lavage.

\section{Microbiological methods}

MICs from recovered pathogens were determined by use of standard National Committee for Clinical Laboratory Standards microtitre MIC methods [11].

\section{Drug measurement}

Plasma concentrations of $\beta$-lactams and fluoroquinolones were determined by use of sensitive and specific high-performance liquid chromatographic assays, as previously described [12-15]. The levels of serum amikacin were determined via fluorescence 
polarisation immunoassay in a Cobas Integra 400 analyser (Roche Diagnostics, Basel, Switzerland), using reagents from the same manufacturer, with a limit of detection of $0.3 \mathrm{mg} \cdot \mathrm{L}^{-1}$.

\section{Definition of clinical and microbiological success and failure}

Clinical success, the primary end-point, was defined as the absence or improvement of clinically significant symptoms and signs such that no additional therapy was required. Clinical failure was defined as the persistence or progression of symptoms and signs or death of the patient.

Microbiological success was defined as eradication or presumed eradication (for patients assessed as cured if no specimens were obtained) of all pathogens isolated at baseline. Non-eradication was defined as persistence or presumed persistence (if no sputum sample was available for a case classified as "clinical failure").

\section{Statistical methods}

Patients with both pathogen MIC determination and drug concentration measurement were the intervention group. All other patients were the control group. Categorical variables were compared by the Pearson Chi-squared or Fisher's exact test. Continuous variables were compared by unpaired t-test or Mann-Whitney U-test. For comparisons involving more than two groups, the Pearson Chi-squared test was used for categorical variables; ANOVA or the Kruskal-Wallis test was used for continuous variables. Breakpoints in the distribution of continuous variables were determined by Classification And Regression Tree (CART) analysis, a statistical tool to identify breakpoints within a continuous variable where the outcome of interest is distinctly different between the resulting groups.

\begin{tabular}{lccc}
\hline $\begin{array}{l}\text { TABLE 1 } \\
\text { Characteristic }\end{array}$ & Evaluated patients & Controls & p-value \\
\hline & $205(117 / 88)$ & $433(247 / 186)$ & NS \\
Patients (M/F) $\mathbf{n}$ & $67 \pm 8(41-86)$ & $69 \pm 8(41-86)$ & NS \\
Age yrs & & & \\
Diagnosis & $172(83.9)$ & $415(95.84)$ & $<0.001$ \\
NP & $33(16.1)$ & $18(4.16)$ & \\
NP with bacteraemia & $17.8 \pm 5.0$ & $19.02 \pm 4.6$ & NS \\
APACHE II score & $(10-32)$ & $(10-30)$ & \\
& & & \\
Antibiotics & $90(24.7)$ & $224(28.35)$ & \\
Ceftazidime & $90(24.7)$ & $140(17.73)$ & \\
Cefotaxime & $87(23.9)$ & $208(26.32)$ & \\
Levofloxacin & $56(15.4)$ & $147(18.6)$ & \\
$\quad$ Ciprofloxacin & $41(11.3)$ & $71(9.0)$ & \\
Amikacin & $364(100)$ & $790(100)$ & \\
Total & $159(77.6)$ & $261(78.4)$ & NS \\
Antibiotic combination & & & \\
therapy & &
\end{tabular}

Data are presented as mean \pm SD (range) or $n(\%)$, unless otherwise stated. M: male; F: female; NP: nosocomial pneumonia; APACHE: Acute Physiology and Chronic Health Evaluation; NS: nonsignificant.
Logistic regression was employed for analysis of dichotomous outcomes. For univariate analyses, all covariates that differed between treatment groups $(\mathrm{p} \leqslant 0.2)$ were considered for model entry in the multivariate analysis. The variable with the greatest log-likelihood was entered into the model first, and the likelihood ratio test was used to determine the appropriateness of model expansion. This metric was defined as twice the log-likelihood difference between the base and the expanded models evaluated against a Chi-squared distribution with the appropriate number of degrees of freedom. The $\mathrm{p}$ value criterion for expansion was $<0.05$. SYSTAT for Windows v. 11.0 (Systat Software,Chicago, IL USA) was employed for all statistical analysis. Values of $p \leqslant 0.05$ were considered statistically significant.

\section{RESULTS}

\section{Patient characteristics}

A total of 638 patients with NP were enrolled in this study. In 205 patients, both drug concentration and infecting microrganism MIC values were available, while in the other 433 patients who were used as controls, one or both parameters were lacking. 24 patients in the test group and 52 in the control group were intubated for $<5$ days at the time of study entry. Table 1 shows the baseline characteristics of the two groups. Average age and APACHE II score were similar in both the test and control groups: $67 \pm 8$ (range $41-86$ ) versus $69 \pm 8$ yrs (range 41-86) and 17.8 \pm 5.0 (10-32) versus $19.02 \pm 4.6$ (10-30), respectively. Ceftazidime and cefotaxime were the most frequently utilised antibiotics in the treatment of NP. Antibiotic combination therapy was utilised in $77.6 \%$ of cases in the test group and in $78.4 \%$ in the control group (table 1).

\section{Isolated organisms}

In the first group, a total of 205 pathogens were isolated; the most frequent was Streptococcus pneumoniae (45 isolates), followed by Haemophilus influenzae (32 isolates), Pseudomonas aeruginosa (29 isolates), Klebsiella spp. (25 isolates), Enterobacter spp. (24 isolates), Proteus spp. (15 isolates), Escherichia coli (14 isolates) and Serratia spp. (13 isolates). Among Gram-negative organisms, the most frequently isolated species were $P$. aeruginosa (14.1\% of all isolates). In the control group, only 142 pathogens were isolated: S. pneumoniae (34 isolates), $P$. aeruginosa (25 isolates), H. influenzae (24 isolates), Klebsiella spp. (18 isolates), Enterobacter spp. (15 isolates), Proteus spp. (seven isolates), E. coli (seven isolates), Serratia spp. (10 isolates) and Citrobacter (two isolates). Table 2 shows the MIC range of all isolates from the test group.

\section{Therapeutic outcomes}

Success rates and overall death rate among patients in the PK/ PD evaluated group, as well as the length of stay, was significantly better than in the control group. However, the duration of mechanical ventilation was not statistically different (table 3). For clinical outcomes, the APACHE II score, combination chemotherapy and measurement of MIC and drug exposure with subsequent decision regarding drug dose/ schedule alteration (or not) had a significant impact on the probability of a good clinical outcome. The final model is shown in table 4 . The impact of the covariates on the probability of clinical outcome is displayed in figure 1. 


\begin{tabular}{|c|c|c|c|c|c|c|}
\hline & \multirow[t]{2}{*}{ Isolates $\mathrm{n}$} & \multicolumn{5}{|c|}{ MIC $\mathrm{mg} \cdot \mathrm{L}^{-1}$} \\
\hline & & Ceftazidime & Cefotaxime & Levofloxacin & Ciprofloxacin & Amikacin \\
\hline S. pneumoniae & 45 & $1-2$ & $0.03-1$ & $0.03-2$ & $2-8$ & \\
\hline Klebsiella spp. & 25 & $0.12-8$ & $0.06-4$ & $0.03-2$ & $0.03-2$ & $0.03-4$ \\
\hline Enterobacter spp. & 24 & $0.03-2$ & $0.03-1$ & $0.03-1$ & $0.03-1$ & $0.03-2$ \\
\hline Proteus spp. & 15 & $0.03-2$ & $0.03-2$ & $0.03-1$ & $0.5-1$ & $0.12-1$ \\
\hline E. coli & 14 & $0.06-1$ & $0.03-2$ & $0.06-2$ & $0.03-1$ & 1 \\
\hline Serratia spp. & 13 & $0.06-4$ & $0.03-4$ & $0.12-2$ & $0.5-1$ & $0.12-4$ \\
\hline
\end{tabular}

Data are presented as ranges, unless otherwise stated. S. pneumoniae: Streptococcus pneumoniae; H. influenzae: Haemophilus influenzae; P. aeruginosa: Pseudomonas aeruginosa; E. coli: Escherichia coli.

For microbiological outcome, the MIC of the infecting pathogen for the $\beta$-lactams and whether the second drug was a fluoroquinolone or aminoglycoside affected the probability of eradication (better outcomes when the $\beta$-lactam MIC was $\leqslant 2 \mathrm{mg} \cdot \mathrm{L}^{-1}$ and with the fluoroquinolone). Adjusting dose also significantly affected eradication probability. Table 5 shows the final model for microbiological outcome, which was the secondary end-point.

All treatments were well tolerated, no differences in sideeffects were found between groups.

\section{DISCUSSION}

Recently, numerous studies in vitro and in animal infection models have been performed to elucidate the correlation between antimicrobial therapeutic efficacy and the PK/PD indices of antimicrobials, such as the $t>\mathrm{MIC}$, the ratio of the 24-h AUC to the MIC (AUC24h/MIC ratio), and the Cmax related to the MIC ( $\mathrm{Cmax} / \mathrm{MIC}$ ratio). The general view is that $t>\mathrm{MIC}$ is the major PK/PD index that determines the in vivo efficacy of $\beta$-lactams, including penicillins, cephalosporins, monobactams and carbapenems, while, conversely, the Cmax/ $\mathrm{MIC}$ and AUC24h/MIC ratios are the important PK/PD indices that correlate with the efficacy of aminoglycosides and fluoroquinolones $[6,7,16,17]$.

In this study, we have employed the idea of knowledge of the PD-linked variable and a target value for this variable to test the hypothesis that outcome could be improved by identifying patients early in their infectious course that are probably undertreated (i.e. measured drug exposure, relative to the pathogen MIC, is below the target value). Indeed, this is the central hypothesis of this investigation. We also wished to determine whether there was an impact on microbiological eradication. We felt that studying a specific infectious indication would allow a better test of the hypothesis and, therefore, we restricted our study to early and late NP.

Indeed, measuring drug exposure and determining the organism MIC allowed adjustment of dose in 81 out of 205 patients. Making the judgement that therapy was adequate or rapidly adjusting dose in the first 3 days of therapy was one of the factors that had a positive impact on obtaining a good clinical outcome for these patients, relative to the group of patients where either the MIC or the drug exposure was unavailable. Figure 1 demonstrates the impact of each of the covariates shown to have a significant impact on the

TABLE 3 Treatment success rates and mean length of stay

\begin{tabular}{lccc} 
& Evaluated patients & Controls & p-value \\
\hline Patients $\mathbf{n}$ & 205 & 433 & \\
Cure $\mathbf{n}$ & 168 & 293 & $<0.001$ \\
Failure & $37(18.04)$ & $140(32.33)$ & $<0.001$ \\
Mortality or AMA & $21(10.24)$ & $14.86 \pm 3.94$ & 0.0076 \\
Length of stay days & $12.35 \pm 3.62$ & $5.39 \pm 1.8^{*}$ & 0.09 \\
Duration of mechanical ventilation days & $4.28 \pm 1.3^{\#}$ & \\
\hline
\end{tabular}

Data are presented as $n(\%)$ or mean \pm SD, unless otherwise stated. AMA: patients left hospital against medical advice. ${ }^{*}: \mathrm{n}=25 ;{ }^{\bullet}: \mathrm{n}=52$. 


\begin{tabular}{|c|c|c|c|c|}
\hline TABLE 4 & $\begin{array}{l}\text { model } p \\
\text { nts with }\end{array}$ & $\begin{array}{l}\text { rameters } \\
\text { ssocomi }\end{array}$ & $\begin{array}{l}\text { or clinical } \\
\text { pneumon }\end{array}$ & utcome for \\
\hline Parameter & Estimate & $p$-value & Odds ratio & $95 \% \mathrm{Cl}$ \\
\hline Constant & 4.425 & $<0.0001$ & & \\
\hline Monotherapy & 1.208 & 0.0014 & 3.349 & $1.592-7.043$ \\
\hline Adjustment ${ }^{\top,+}$ & 0.8661 & 0.0002 & 2.238 & $1.514-3.735$ \\
\hline APACHE II score & -0.1930 & $<0.0001$ & 0.8245 & $0.7874-0.8633$ \\
\hline
\end{tabular}

$\mathrm{Cl}$ : confidence interval; APACHE: Acute Physiology and Chronic Health Evaluation. ${ }^{*}: \mathrm{n}=638$; " : two-level categorical covariates; ${ }^{+}$: includes patients who had minimum inhibitory concentration determined and drug exposure measured and were determined to be adequately treated or who had their doses/schedules adjusted; ${ }^{\text {s: }}$ : continuous covariate.

probability of a good clinical outcome. The most important variable was the APACHE II score (table 4), which is clinically believable. As a measure of the other factors, we calculated the APACHE II score at which the probability of a good clinical outcome fell below $90 \%$. This APACHE II score was 22 in the group measured/adjusted and treated with monotherapy and 16 in the measured/adjusted group treated with combination

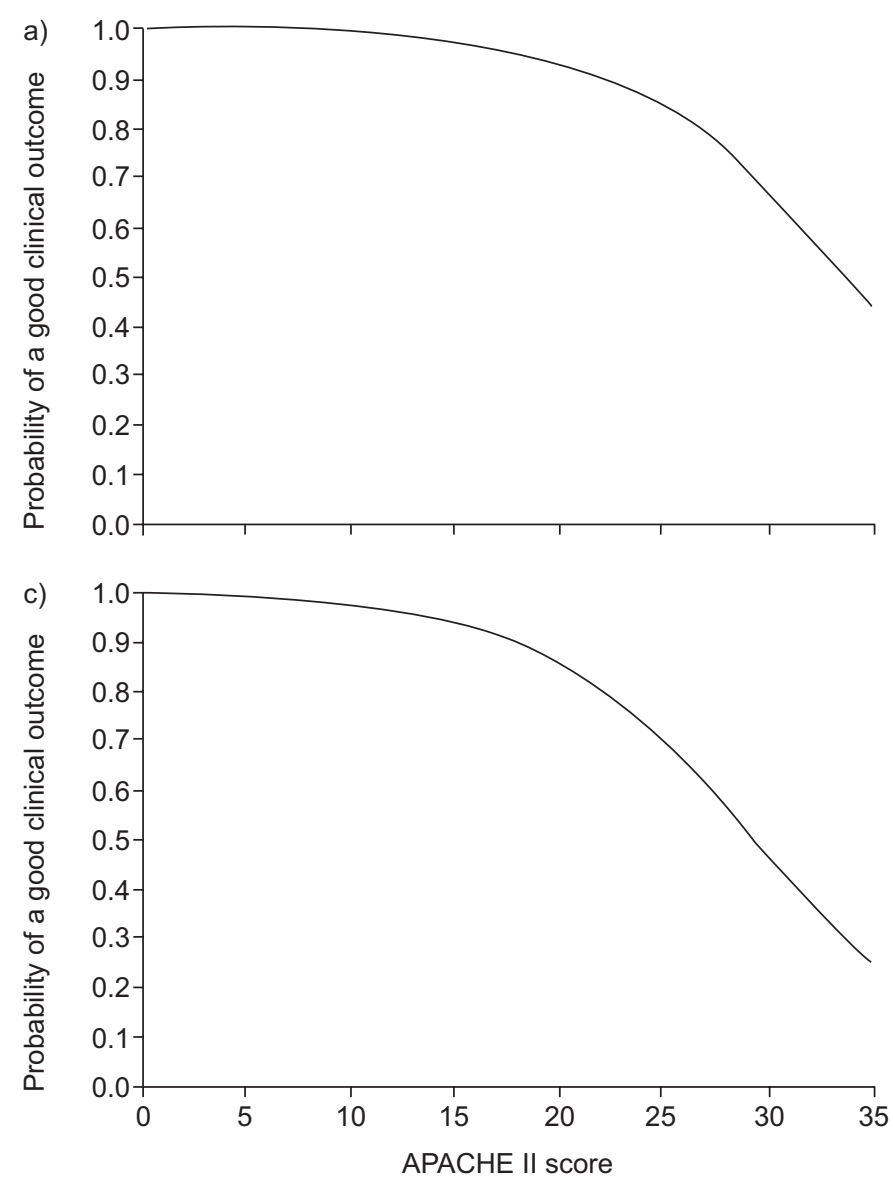

\begin{tabular}{|c|c|c|c|c|c|}
\hline \multirow{2}{*}{$\begin{array}{l}\text { TABLE } 5 \\
\text { Parameter }\end{array}$} & \multicolumn{5}{|c|}{$\begin{array}{l}\text { Final model for microbiological outcome for } \\
\text { patients with nosocomial pneumonia\# }\end{array}$} \\
\hline & & Estimate & $p$-value & Odds ratio & $95 \% \mathrm{Cl}$ \\
\hline Constant & & 2.375 & $<0.0001$ & & \\
\hline Amikacin & & -0.8968 & 0.0177 & 0.4079 & $0.1944-0.8556$ \\
\hline$\beta$-lactam MIC & & -2.266 & $<0.0001$ & 0.1037 & $0.0502-0.2143$ \\
\hline $\begin{array}{l}\text { Measure/no } \\
\text { adjustment }\end{array}$ & & -0.255 & $0.5375^{\circ}$ & 0.7751 & $0.3450-1.7452$ \\
\hline Measure/adjus & stment & 1.1276 & 0.0207 & 3.088 & $1.188-8.029$ \\
\hline
\end{tabular}

All covariates are categorical with two levels, except for measure/adjustment, which has three levels: measure/no adjustment, measure/adjustment and no measure/no adjustment. Cl: confidence interval; MIC: minimum inhibitory concentration. ${ }^{*}: \mathrm{n}=272 ;{ }^{\bullet}$ : nonsignificant.

therapy. For the non-measured, nonadjusted group, these values were 18 (versus 22) and 12 (versus 16), respectively.

It may seem odd that the monotherapy group performed better than the combination therapy group. One may hypothesise antagonism or, perhaps, excess toxicity. However, the likeliest explanation arises from the pathogens being treated in each

b)

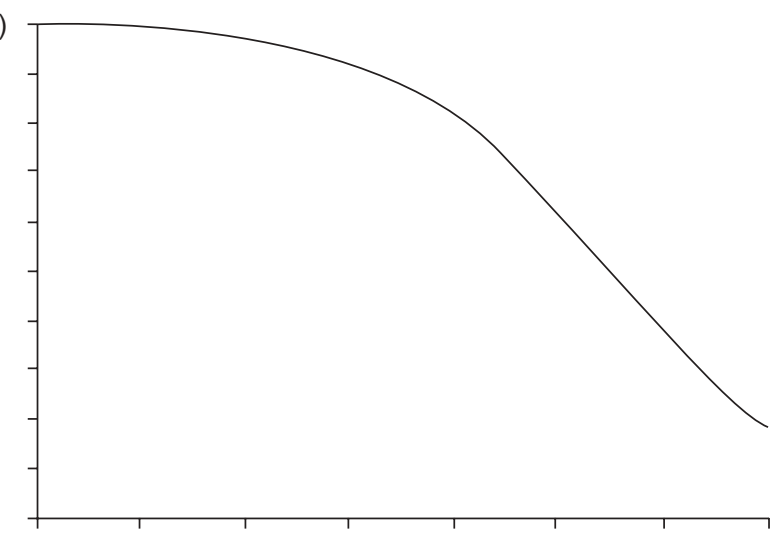

d)

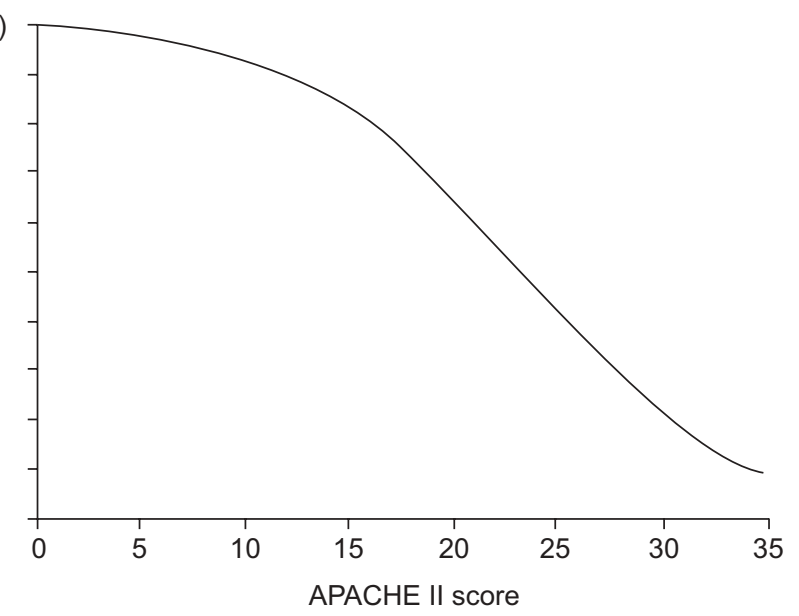

FIGURE 1. Impact of $a$ and b) adjusting therapy versus $c$ and d) no dose adjustment, or use of a and c) monotherapy versus $b$ and d) combination therapy on the probability of a good clinical outcome, as a function of Acute Physiology and Chronic Health Evaluation (APACHE) II score. 
group. Of the 69 pathogens recovered from the monotherapy group, $59(86 \%)$ isolates were either $H$. influenzae or $S$. pneumoniae, with only nine isolates of Enterobacteriaceae and one $P$. aeruginosa isolate. Among the 278 isolates from the combination therapy group, $76(27 \%)$ were $H$. influenzae or $S$. pneumoniae, while 145 (52\%) were Enterobacteriaceae, 53 (19\%) were $P$. aeruginosa and four $(1.4 \%)$ were Stenotrophomonas maltophilia. The combination therapy group was populated by more difficult to treat nosocomial Gram-negative pathogens, which probably explains the result identified.

It is important to note that we also analysed the impact of measuring and not adjusting versus measuring and adjusting versus not measuring and not adjusting (data not shown). This three-level categorical variable was also significant (with the same other covariates) and demonstrated that those measured and adjusted actually had the best outcomes. We may conclude that the primary hypothesis was validated and that measurement of drug exposure and early identification of the causative pathogen can identify patients at high risk for a poor clinical outcome and, perhaps most importantly, that early (first 3 days) therapeutic intervention can result in a better clinical outcome.

Secondarily, we also wished to examine microbiological outcome. Here, adjustment also had a positive impact on outcome (table 5). The other covariates were having a low MIC for the $\beta$-lactam being employed $\left(\leqslant 2 \mathrm{mg} \cdot \mathrm{L}^{-1}\right)$ and the use of a fluoroquinolone versus an aminoglycoside.

In this instance, it is clear that only the patients with measured drug exposure and MIC with dose/schedule alteration had a significantly higher rate of eradication. This is not overly surprising, as alteration of dose/schedule tended to place patients far away from the breakpoints, whereas a fraction of patients had values near the breakpoints but were not dose/ schedule altered.

Having a low $\left(\leqslant 2 \mathrm{mg} \cdot \mathrm{L}^{-1}\right)$ MIC for the $\beta$-lactams employed here is also quite concordant with our understanding of antimicrobial chemotherapy, as both drugs will have high target attainment rates at these lower MIC values at the doses and schedules employed. The raw eradication rate for isolates with MIC values $\leqslant 2 \mathrm{mg} \cdot \mathrm{L}^{-1}$ and greater than this value was $190(90 \%)$ out of 210 versus 37 (53\%) out of 70 .

Somewhat surprising was the finding that use of a fluoroquinolone had a significant impact on sterilisation. Aminoglycosides achieved sterilisation in 41 (65\%) out of 63 instances, compared with $224(88 \%)$ out of 255 instances for fluoroquinolones. It should also be noted that aminoglycosides were never administered alone, while fluoroquinolones were administered alone in 45 patients. In $36(80 \%)$ out of 45 patients, the infecting pathogen was either $H$. influenzae or $S$. pneumoniae, where, particularly for levofloxacin, MIC values are quite low for these pathogens and eradication would be expected. Even in combination therapy, 71 (33\%) out of 213 patients had these pathogens treated with the fluoroquinolone, whereas the aminoglycoside was employed in combination in 65 patients, of whom 51 (78\%) were infected with $P$. aeruginosa, Enterobacter spp., Klebsiella spp. or Serratia spp. These hospitalacquired pathogens make the low eradication rate somewhat understandable.
In summary, we tested a simple hypothesis. Understanding antimicrobial pharmacodynamics allows choice of therapeutic targets. Measuring drug exposure and the pathogen MIC allows a judgement to be made as to whether dose and schedule need to be altered. We recognised the importance of making such an intervention quickly. Consequently, in this study, patients had blood obtained for drug measurement early in the course, so that both pieces of information were available in the same time frame. The data demonstrated that making therapy adequate, as judged by attaining the prospectively set therapeutic targets or altering dose and schedule early in the clinical course to hit these targets, resulted in significantly better outcomes. Such interventions should be trialled again, both in pneumonia as well as in other therapeutic indications.

\section{SUPPORT STATEMENT}

This study was supported by a grant from the Italian Ministry of Health (No. 2003051858; Rome, Italy).

\section{STATEMENT OF INTEREST}

None declared.

\section{REFERENCES}

1 American Thoracic Society, Infectious Diseases Society of America, Guidelines for the management of adults with hospitalacquired, ventilator-associated, and healthcare-associated pneumonia. Am J Respir Crit Care Med 2005; 171: 388-416.

2 Chastre J, Fagon JY. Ventilator-associated pneumonia. Am J Respir Crit Care Med 2002; 165: 867-903.

3 Heyland DK, Cook DJ, Griffith L, et al. The attributable morbidity and mortality of ventilator-associated pneumonia in the critically ill patient. The Canadian Critical Trials Group. Am J Respir Crit Care Med 1999; 159: 1249-1256.

4 Kollef $\mathrm{MH}$. Inadequate antimicrobial treatment: an important determinant of outcome for hospitalized patients. Clin Infect Dis 2000; 31: Suppl. 4, S131-S138.

5 Scaglione F, Paraboni L. Influence of pharmacokinetics/pharmacodynamics of antibacterials in their dosing regimen selection. Expert Rev Anti Infect Ther 2006; 4: 479-490.

6 Drusano GL, Johnson DE, Rosen M, et al. Pharmacodynamics of a fluoroquinolone antimicrobial agent in a neutropenic rat model of Pseudomonas sepsis. Antimicrob Agents Chemother 1993; 37: 483-490.

7 Drusano GL. Antimicrobial pharmacodynamics: critical interactions of "bug and drug". Nat Rev Microbiol 2004; 2: 289-300.

8 Scaglione F. Can PK/PD be used in everyday clinical practice. Int J Antimicrob Agents 2002; 19: 349-353.

9 Preston SL, Drusano GL, Berman AL, et al. Levofloxacin population pharmacokinetics and creation of a demographic model for prediction of individual drug clearance in patients with serious community-acquired infection. Antimicrob Agents Chemother 1998; 42: 1098-1104.

10 Forrest A, Ballow CH, Nix DE, et al. Development of a population pharmacokinetic model and optimal sampling strategies for intravenous ciprofloxacin. Antimicrob Agents Chemother 1993; 37: 1065-1072.

11 National Committee for Clinical Laboratory Standards (NCCLS)., Methods for Dilution Antimicrobial Susceptibility Tests for Bacteria That Grow Aerobically. NCCLS document M2-A7. Wayne, NCCLS, 2000.

12 Wong FA, Juzwin SJ, Flor SC. Rapid stereospecific high-performance liquid chromatographic determination of levofloxacin in human plasma and urine. J Pharm Biomed Anal 1997; 15: 765-771. 
13 Mack G. Improved high-performance liquid chromatographic determination of ciprofloxacin and its metabolites in human specimens. J Chromatogr 1992; 582: 263-267.

14 Kraemer HJ, Gehrke R, Breithaupt A, et al. Simultaneous quantification of cefotaxime, desacetylcefotaxime, ofloxacine and ciprofloxacine in ocular aqueous humor and in plasma by highperformance liquid chromatography. J Chromatogr B Biomed Sci Appl 1997; 700: 147-153.
15 Hanes SD, Herring VL, Wood GC. Alternative method for determination of ceftazidime in plasma by high-performance liquid chromatography. J Chromatogr B Biomed Sci Appl 1998; 719: 245-250.

16 MacGowan AP. Role of pharmacokinetics and pharmacodynamics: does the dose matter? Clin Infect Dis 2001; 33: Suppl. 3, 238-239.

17 Barger A, Fuhst C, Wiedemann B. Pharmacological indices in antibiotic therapy. J Antimicrob Chemother 2003; 52: 893-898. 